\title{
ANTIPROTON YIELD DIAGNOSTICS FOR THE TEVATRON I DEBUNCHER
}

\author{
C.D. Johnson, CERN \& C. Hojvat, Fermilab
}

October 17,1984

\section{INTRODUCTION}

During start-up of the CERN AA, many hours of machine experiments went into the study and optimization of antiproton yields. Those involved in the commissioning programme experienced the difficulty of tuning a new machine to accept a low-intensity full-aperture beam. The antiproton yield could only be obtained by integrating a slow schottky scan of the beam on the injection orbit, normalized with respect to primary beam intensity by a charge transformer just in front of the production target. A precise yield measurement took about five minutes. At high yields this method permitted measurements to within a few percent. The slowness of the multi-parameter yleld optimization, starting from low ylelds where the measurement errors were often as large as the gains to be made, cannot be over emphasized.

In the Tevatron I Debuncher the antiproton yields should be substantially higher than at the AA and, given a schottky pick-up of sufficient sensitivity, the situation looks more promising. At the AA we have resolved some of our difficulties by improving the charge transformer signal, speeding up the Schottky scan and adding instrumentation to use the signals from pions, muons and electrons injected along with the antiprotons. Low yields, e.g. at reduced aperture, are now measured using beam scrapers in conjunction with counters calibrated against the Schottky pick-up at high intensities. The latter is itself calibrated by the circulating beam current transformer at even higher intensities, usually with protons in reverse polarity mode.

Based on the AA experience we outline the techniques that could be used for the following measurements and procedures at the Debuncher:

1) antiproton yield (number of antiprotons circulating in the Debuncher per incident proton) versus the machine apertures $\Delta x, \Delta y$, and $\Delta p$,

ii) yleld versus phase space coordinates downstream from the production target,

iii) use of other secondary particle fluxes, 
iv) optimization of full-aperture yield at the start of and during antiproton accumulation,

\section{DIAGNOSTIC METHODS AND INSTRUMENTATION}

\section{$2.1 \quad 120 \mathrm{GeV}$ Proton Beam Line, Ap-1}

A beam current transformer just before the target will be used for proton beam intensity normalization. It should have an accuracy of the order of 3\% over its dynamic range. Other transformers in the line will be used for beam line transmission efficiency. They do not enter into the antiproton yield measurement, except as a check on the stability of the target transformer. We expect measurements to be made over two orders of magnitude of proton intensity $\left(10^{10}\right.$ to $10^{12}$ protrons per $1.6 \mu$ s pulse).

Proton beam position and size at the target will be monitored using high resolution SEM grids located just before the target. At CERN an electrostatic plck-up upstream of the target and a SEM grid at the target are available, but noise problems have not yet been eliminated. The only monitor regularly used is a fluorescent screen with a $2 \mathrm{~mm}$ diameter hole incorporated upstream of the target assembly. Beam size information is obtained from scans of yleld versus beam position at the target, as measured on the screen. Yield versus beam size can be studied by reducing the beam emittances in the PS. The present instrumentation is minimal, and both Fermilab and CERN could benefit from the development of suitable SEM grids. A joint effort between CERN and Fermilab is underway.

The present Fermilab plan for proton beam measurements includes one pair of $x-y$ SEM wire chambers with wire spacing of $0.25 \mathrm{~mm}$ in the beam central region. The expected resolution on the beam width at the target is $0.10 \mathrm{~mm}$ rms. Furthermore, two electrostatic beam position detectors should give the relative beam position at the target with an error of less than $0.10 \mathrm{~mm}$ rms.

\subsection{Antiproton Beam Line (Target to Debuncher), AP-2}

Beam transformers, SEM grids and screens in the antiproton line are of dubious value except for reverse ejection of protons from the Debuncher up to the target. During the lithium lens tests at CERN in 1983, use was made of the pion plus electron flash on injection screens (significantly more visible than during normal horn operation).

A beam transformer downstream from the target could be used as a crude operational monitor of the total number of interactions in the target. This number is sensitive to the proton beam position and size at the target, the target integrity, etc. 
The acceptance of the antiproton beam line should be measured at an early stage. Unless one relies on the measurement of the total secondary particle flux, the Debuncher must be used as an storage ring to distinguished antiprotons from other decaying particles. The accuracy of this method depends on the understanding of the soft and hard aperture limits in the Debuncher. An alternative is to select a region in phase space using antiproton beam line collimators and inject this collimated beam always onto the central orbit of the Debuncher for measurement.

The antiproton injection line (AP2) is well suited for the instalation of collimators. Momentum collimation in the high dispersion region (left bend) and pairs of transverse collimators in the regular FODO section before the left bend, separated by $\pi / 2$ phase advance, would permit the study of the antiproton distribution in phase space. This is particularly important to achieve maximum filling of the Debuncher aperture. The collimators should be designed such that each jaw can be moved independently, thus permitting scanning the apertures with a slit. If equipped with steel jaws the length should be 30 to $50 \mathrm{~cm}$. A detailed study of the contribution of interactions, energy degradation and multiple scattering in the collimator's jaws is needed to optimise their design. A preliminary choice of locations within the AP2 beam line is given in the summary.

\subsection{Debuncher Ring}

Beam Transformer

Unless the transformer can measure to an accuracy of a few percent down to beam currents of $50 \mathrm{nA}(1 \%$ of the nominal injected antiproton beam), it is best regarded as an instrument to be used for calibration of a Schottky pick-up and scraper/counter systems. For this purpose it need not be a true DC device. The present Fermilab plan calls for the installation in the Debuncher ring of a $D C$ beam current transformer similar in design to the one installed in the AA Ring at CERN. The AA has achieved by this method an accuracy of $\pm 10^{s} \bar{p}^{\prime s}$ on their measurements. (Reference 1)

\section{Schottky Pick-Up}

A calibrated Schottky pick-up permits measurement of total yield, momentum aperture and yleld versus momentum. In the Debuncher the sum signal from the betatron cooling pick-ups will be avallable for this purpose. Sensitivity requirements have been mentioned previously. The signal band used in the measurement will be chosen empirically at some harmonic of the revolution frequency which gives maximum response. A fast measurement of yield under fixed momentum aperture conditions can be obtained by setting the spectrum analyser at the center frequency of the band with suitably wide bandwidth. 
Transverse Aperture

Measurements of the Debuncher transverse acceptance could be done with protons. Filling of the aperture will nevertheless require using the injection and transfer kickers to produce coherent betatron oscillations, as is done at the $A A$. In the Debuncher we have the possibility of exploring both vertical and horizontal apertures using the two kickers (injection and extraction). Provision must be made to have variable amplitude asynchronous kicks on request.

Beam scrapers, and an array of particle counters, should be installed in a zero dispersion region for studies of antiproton yields (or proton intensity) versus transverse aperture. Although the calculated antiproton yield increases with the square of the acceptance at low acceptances, and follows a linear relationship at large acceptances (see e.g. reference 2), in the AA it falls off much more rapidly due to the loss of particles with simultaneous large $x$ and large $y$ coordinates. There is up to a factor of two in yield to be made up by fully populating the outer regions. A mechanism permitting exploration of the corners of the aperture, utilizing horizontal and vertical scrapers, would be useful to study this region of phase space. Amplitude distributions can be obtained by scaling the output from the counters into a multi-channel scaler as a scraper is driven into the beam. Real-time position information and computing power is required. At the AA a LRS 3500 micro- computer with interactive graphics is used for this purpose.

The location of the antiproton scraper to measure transverse acceptances is fairly flexible provided that a zero momentum dispersion region is chosen to separate the transverse and longitudinal phase spaces. The location proposed in Figure 2, straight section "D206" just downstream of $D 2 Q 7$, is in a region where the horizontal and vertical $\beta$ values are each $8 \mathrm{~m}$, thus the maximum beam half-size in each plane $1 \mathrm{~s} 13.5 \mathrm{~mm}$.

\section{Longitudinal Acceptance}

of the flux of secondary particles injected into the Debuncher together with the antiprotons, electrons will remain circulating for a number of turns. It can be assume that the electrons will have the same longitudinal distribution as the antiprotons at production and that this distribution will be modified by the beam transport such that it mimics the antiproton distribution injected and accepted by the Debuncher.

The use of electrons for injection tuning and for the study of the longltudinal acceptance, relies on their energy loss due to synchrotron radiation. Electrons spiraling into inner aperture limits just after injection have been used at the AA for studies of longitudinal acceptances, injection tuning and as an operational yield monitor 
(Reference 1). In the AA the precooling shutters are convenient aperture stops that intercept the electrons at the inner radius of the machine.

The observed electron loss rate on a scraper is related to the rate at which the electron's orbit radius decreases due to synchrotron radiation. The relevant loss of energy is for the electrons at the lower end of the momentum acceptance of the machine, where the scraper is to be located. Any coherent betatron motion in the horizontal plane will modulate the rate at whlch electrons fall onto the scraper. Coherent betatron motion could be induced by a variety of reasons, including injection errors. As the loss of electrons on the scraper is expected to be easily detectable, a sensitive tool is available for tunning injection or studying other orbit errors. We will assume that, although more than one scraper could be present, only one scraper presents a real restriction into the machine aperture. We will also assume that the distribution of electrons in momentum is uniform, that It is not exhausted for many machine revolutions and that it is not distorted by the different synchrotron radiation rates within the momentum acceptance of the machine. If more than one scraper is located to form an aperture restriction, the gap left in the electron distribution will be repopulated by the radiative losses between scraper locations. In this case only the arbitrary normalization introduced below will be affected. The actual loss rate at any one scraper will depend in amplitude and phase on the relative loss rates at other scrapers and on the betatron phase angle between scrapers. The latter should be chosen to avold integral values of betatron wavelength between succesive scrapers since this would reduce the sensitivity to conerent oscillations of loss rates measured at the downstream scraper. In addition, close orbit changes affect the relative loss rates between scrapers and thus the phase of the loss signal at a particular scraper. (This is thought to account for observed changes in the calibration needed when using this signal for automatic control of injection at the CERN's AA ring). A possibility is to insert the 'active' scraper further into the machine aperture during the coherent oscillation correction excerclse. In the case of the AA ring, the 'scrapers' are the precooling shutters and its position can not be varied.

At a fixed location $(\theta)$ around the Debuncher ring, e.g. the scraper position, the incremental change per turn of the inner electron orbit radial position due to synchrotron radiation is given by:

$$
\Delta x(\theta)=\eta(\theta) \Delta E / E
$$

where $\eta(\theta)$ is the horizontal dispersion at the scraper position and $\Delta \mathrm{E} / \mathrm{E}$ is the relative energy loss per turn. $\Delta \mathrm{E}$ is given by (Reference 3):

$$
\Delta E=88.5 \times 10^{-3}(E / r)
$$


in MeV per revolution, where $E$ is the energy in GeV and $r$ is the bending radius for the dipoles in meters. For the Debuncher we obtain:

$$
\Delta E=88.5 \times 10^{-3} \frac{(8.72 \mathrm{GeV})^{4}}{17.44 \mathrm{~m}}=29.3 \mathrm{MeV}
$$

which is to be compared to the nominal Debuncher aperture of $\Delta E=356$ MeV. In other words, 13 turns after injection all of the electrons would have left the useful aperture of the Debuncher.

In the presence of a coherent betatron oscillation in the horizontal plane, the amplitude of the motion of the beam centre after $n$ turns at the same location $(\theta)$ in the ring, is given by:

$$
x(\theta)=\sqrt{A B(\theta)}[\cos (2 \pi \nu n+\phi)]
$$

where $v$ is the betatron frequency, $A$ is the area enclosed in phase space by the betatron motion of the beam centroid, $\beta(\theta)$ and $\alpha(\theta)$ are the Twiss parameters describing the betatron phase space at the azimuthal location $\theta$ and $\phi$ is an arbitrary phase for the betatron motion such that for $n=0$ then:

$$
\begin{gathered}
x(\theta)=\sqrt{A B(\theta)}[\cos \phi] \\
\text { or, } \phi=\arccos [x(\theta) / \sqrt{A B(\theta)}] \text { when } n=0
\end{gathered}
$$

From equation (4), the increment in the amplitude between turns $(n-1)$ and $n$ can be shown to be:

$$
\Delta x(\theta)=-2 \sqrt{A B(\theta)} \sin (\pi \nu)[\sin (\pi \nu(2 n-1)+\phi)]
$$

The total loss of electrons per turn $\Delta N(\theta)$ will then be proportional to the sum of equations (1) and (5). This incremental loss can be normalized to the case when no betatron oscillations are present :

$$
\Delta N(\theta)=R \text {; or } \Delta N(\theta)=k n(\theta) \Delta E / E=R \text { for } A=0
$$

where $k$ is a proportionality constant. Then the normalized electron loss per revolution, in the presence of coherent oscillations is :

$$
\Delta N(\theta)=R[1-2 \sqrt{A B(\theta)} \sin (\pi \nu) \sin (\delta) /(n(\theta) \Delta E / E)]
$$

where the angle $\delta$ is given by: 


$$
\delta=\pi \nu(2 n-1)+\phi
$$

At the locations chosen for the scrapers (D210-D410-D610), with $n=1.97 \mathrm{~m}, \beta=13.37 \mathrm{~m}$ and a horizontal tune of $v=9.73$ we obtain:

$$
\Delta N=R(1 \pm 760.3 \sqrt{A})
$$

at the extremes of the oscilatory motion; or a sensitivity in the amplitude of oscillations of $1.73 \times 10^{-8} \mathrm{~m}-\mathrm{rad}$ for a $10 \%$ modulation of the rate of electron losses. At the scraper position this translates in a sensitivity of $0.48 \mathrm{~mm}$ and $68 \mu \mathrm{rad}$ and at Debuncher injection in "D403" translates into a sensitivity of $0.40 \mathrm{~mm}$ and 70 urad.

If no scrapers are installed the electrons will be lost uniformly around the ring. As multiplicity in an $8.9 \mathrm{GeV}$ electron-photon shower is of the order of 1000 , the electrostatic beam position pick-ups will probably be swamped by this signal during the first 20 us after injection. To make best use of the electron loss signal the losses can be localised around the machine by adding extra scrapers. The symmetry of the Debuncher lends itself to the use of elther 3 or 6 scrapers. For this purpose movable inner aperture limits should be installed. If required, circults could be added to the beam pick-up amplifiers immediately downstream from these loss points to gate them off during the time interval of electron losses. Figure 1 is a sketch of possible movable electron scrapers, and in Figure 2 we indicate suitable locations.

Beam Loss Monitors and Particle Detectors

Overall Information on beam losses is best obtained from simple scintillation counters disposed around the machine.

Electron losses will require electron shower detectors designed for 500 to $1000 \mathrm{MeV}$ electrons, the mean energies of electrons and positrons leaving the vacuum chamber after the shower development in two radiation lengths of scraper.

Muons from pion decay are most clearly seen at the AA by $a$ Cerenkov monitor with a lucite radiator. Electrons from muon decay should be treated with a little more respect since, given good energy resolution, the muon precession can be observed and used to measure the magnetic field.

In the Debuncher there will be a moderate degree of longitudinal polarization of the circulating muon beam due to the fact that the injection line provides a momentum selected pion beam from which only those muons decaying in the forward direction with momenta close to that of the pions are accepted. The degree of polarization can be 
further improved by reducing the Debuncher transverse and logitudinal acceptances using scrapers in zero and in nigh dispersion regions. The sp in precession frequency is higher that the revolution frequency and so an electron shower counter with its threshold set to respond only to those electrons from the high energy end of the muon decay spectrum will measure a counting rate which varies in time according to:

$$
R=R_{0} e^{-t / t} \mu\left(1-A \sin \left(\omega_{\mu}+\phi\right)\right.
$$

where $t_{\mu}$ is the dilated muon life time (185 $\mu$ sec in the Debuncher) and $\omega_{\mu}$ is given by:

$$
\omega_{\mu}=(\mathrm{g}-2 / 2) \cdot(\mathrm{e} \overline{\mathrm{B}} / \mathrm{m})
$$

where $\bar{B}$ is the mean magnetic field and $(g-2) / 2$ is the anomalous part of the muon magnetic moment.

In the Debuncher $\bar{B}$ is about $0.37 \mathrm{~T}$ and so the electron counter's modulation frequency due to the muon precession will be:

$$
\omega_{\mu} / 2 \pi=59 \mathrm{kHz}
$$

or a period of 17 usec. Accurate measurement of $\omega_{\mu}$ would provide a good check on the value of $\bar{B}$ and hence beam momentum.

\section{Wire Scanner}

Although most needed for stack and extracted beam emittance measurements in the Accumulator, a wire scanner in the Debuncher could be useful, although one would probably have to resort to the detection of soft radiation in order to have enough sensitivity to use it in a non-destructive mode.

Beam scrapers

If the electrostatic pick-ups planned for the Debuncher are able to measure under all injection conditions, then setting-up will be relatively easy. The AA has no such pick-ups and so the initial setting of injection timing and steering is done on the electron loss signal (ref. 1).

Vertical coherent osoillations can be observed using a corner scraper projecting a few mm further into the horizontal aperture than the horizontal scrapers and situated at a different azimuthal location with simultaneous large horizontal dispersion $n$ and large $\beta$. schematically in the top corner of Figure 1. We propose to locate It $_{\text {t }}$ in straignt secton "D109". 
Examples of electron loss signals at the AA are given in figure 3 where the presence of large amplitude coherent oscillations are shown. In the Debuncher we can expect similar signals. As most of the electrons come from $\pi^{\circ}$ decay we have gathered data on negative pion to antiproton ratios in $\mathrm{pN}$ collisions versus secondary particle laboratory momentum at $x=0$ for primary momenta from 24 to $400 \mathrm{GeV} / \mathrm{c}$. The data is shown in figure 4. Assuming that the neutral pions behave in the same way as negative plons, we can expect about four times less electrons per antiproton in the Debuncher than at the AA.

The electron loss signals will be used to correct injection steering and as a fast yield monitor during production. For the latter purpose frequent calibration against the Schottky system or the transformer is advised.

In the Debuncher the electron spill time will be of the order of 20 us. This corresponds to a momentum distribution (including the wings down to zero emittance) of $5.6 \%$. The first $5 \mu \mathrm{s}$ will be obscured by the pions, but the remaining time will be sufficient to observe 3 complete coherent betatron periods. A convenient way to handle this signal is to digitize the analog output from the shower counter, which should be used in DC mode. An FFT, or simply Fourler integrals of the required harmonics, will give the coefflclents corresponding to the electron yield, the amplitude of the coherent oscillations and the width of the gap in the beam. This application is discussed in Reference 1.

The scrapers will be set so as to present well defined inner aperture limits. In the three-scraper solution the electron beam spirals by $2.4 \mathrm{~mm}$ per scraper per turn $\left(\eta_{x}=2.05 \mathrm{~m}\right.$ and the phase advance $=2 n \pi+\pi / 2)$. However, due to incoherent betatron motion the scrapers w1ll not be fully effective unless they cut into the aperture by about $10 \mathrm{~mm}$. This will cause a loss in total acceptance of about $12 \%$ - which is unacceptable. If the scrapers are controllable remotely, the optimum position can be sought experimentally.

The proposed scraper positions, in straight sections "D210", "D410" and "D610" just downstream from the focussing quadrupole and before the the first missing-magnet straight section, were chosen so that, with scrapers of two radiation lengths, the electrons and positrons are sufficiently degraded to be bent out of the vacuum chamber by the next horizontally defocussing quadrupole. The gammas strike the vacuum chamber at the exit of the next dipole. This is shown schematically (with trajectories for 1,2 \& $4 \mathrm{GeV}$ electrons and positrons) in Figure 5. The choice of $\beta_{x}$ is not important since the ratio $\sqrt{ } \beta_{x} / \eta$ is almost constant in that region of the machine.

The best positions for the shower counters would be at the inner downstream end of the second missing-magnet section, or just beyond the first downstream dipole on the outside of the ring. 
Calculations using the program EGS3 predict for a scraper having a thickness of 2 radiation lengths of iron ( $\mathrm{Fe}$ ) the following shower compostion:

$\begin{array}{lcc} & \text { mean energy (MeV) } & \text { multiplicity } \\ \text { electrons } & 810 & 2.8 \\ \text { positrons } & 540 & 2.0 \\ \text { gammas } & 657 & 6.7\end{array}$

Only $5 \%$ of the electrons have greater than $2 \mathrm{GeV}$ and only $1.2 \%$ have more than $4 \mathrm{GeV}$.

\section{SUMMARY}

The system here described will permit the study of antiproton yield from the target and of that fraction captured into the Debuncher phase space acceptance. Equally, other secondary particles could be used as diagnostic tools for optimization of antiproton yields and tuning of the Debuncher.

The following instrumentation will be required:

1) 1 horizontal collimator with individually adjustable jaws in the antiproton line AP-2 at location "719" for momentum bite selection.

2) 4 sets of collimators with individually adjustable jaws in antiproton line AP-2 at locations "708" and "710" for horizontal collimation and at locations "707" and "709" for vertical collimation.

3) 3 sets of "e--scrapers" at stra1gnt sections "D210" "D410" and "D610".

4) 1 "e--corner scraper" at straight section "D109" towards D1Q10.

5) 1 " $\bar{p}$-scraper" at straight section "D406".

6) 4 sets of shower counters associated with each $e^{-}$- scraper.

7) Counter telescope associated with the $\bar{p}$ scraper.

\section{REFERENCES}

1. Antiproton Field Optimization in the CERN Antiproton Accumulator, C.D. Johnson, IEEE Trans. Nucl. Sc1., NS30 (1983) 2821.

2. Calculations of Antiproton Ylelds for the Fermilab Antiproton Source C. Hojvat and $A$. Van Ginneken, Nuc. Instr. and Meth., 206 (1983) 67.

3. M. S. Livingston and J. P. Blewett, Particle Accelerators, 
McGraw H111, 1962 , p.216.

4. F.J.M. Farley, E. Plcasso, Ann. Rev. Nuclear and Particle Sclence, 29 (1979) 243. 


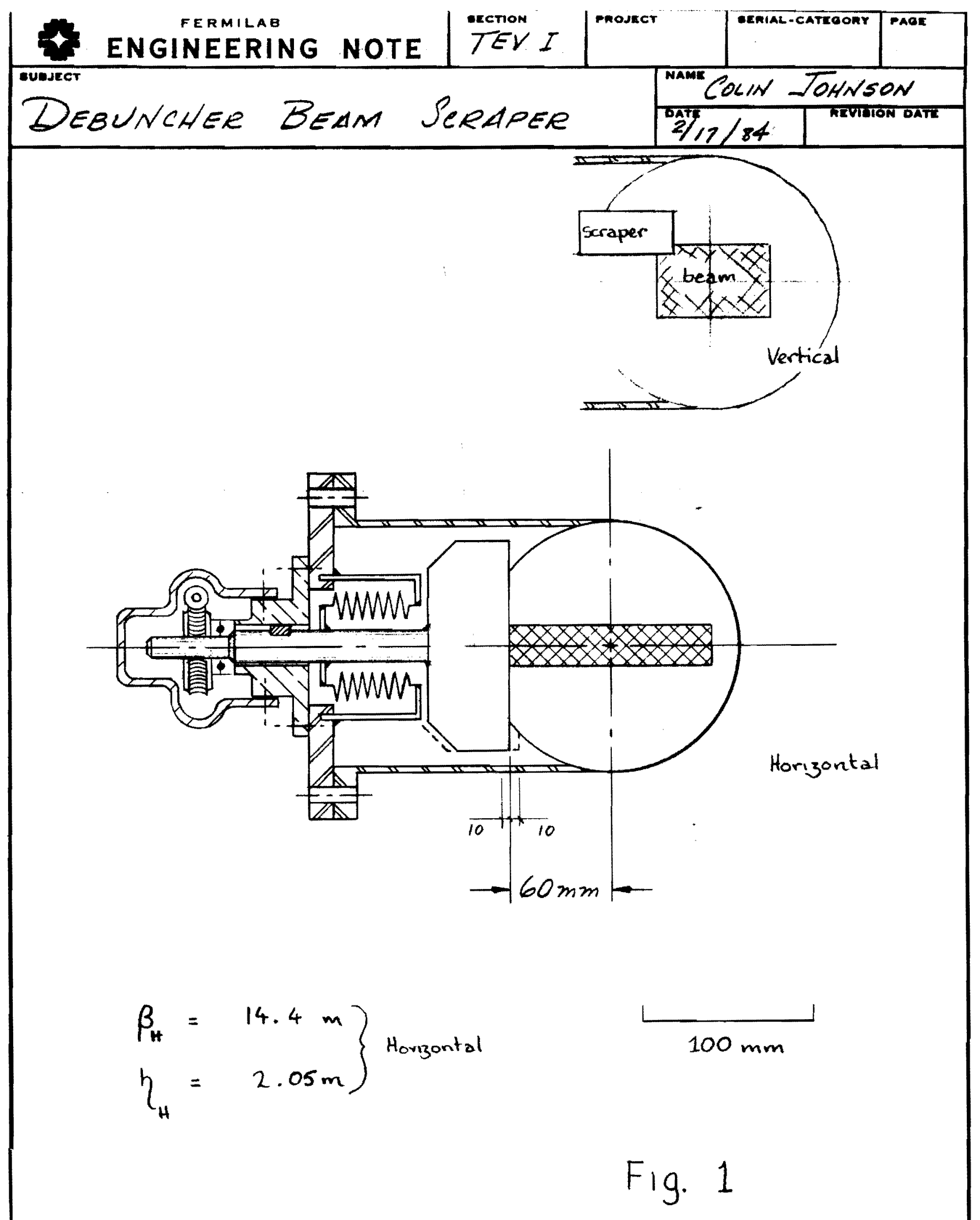




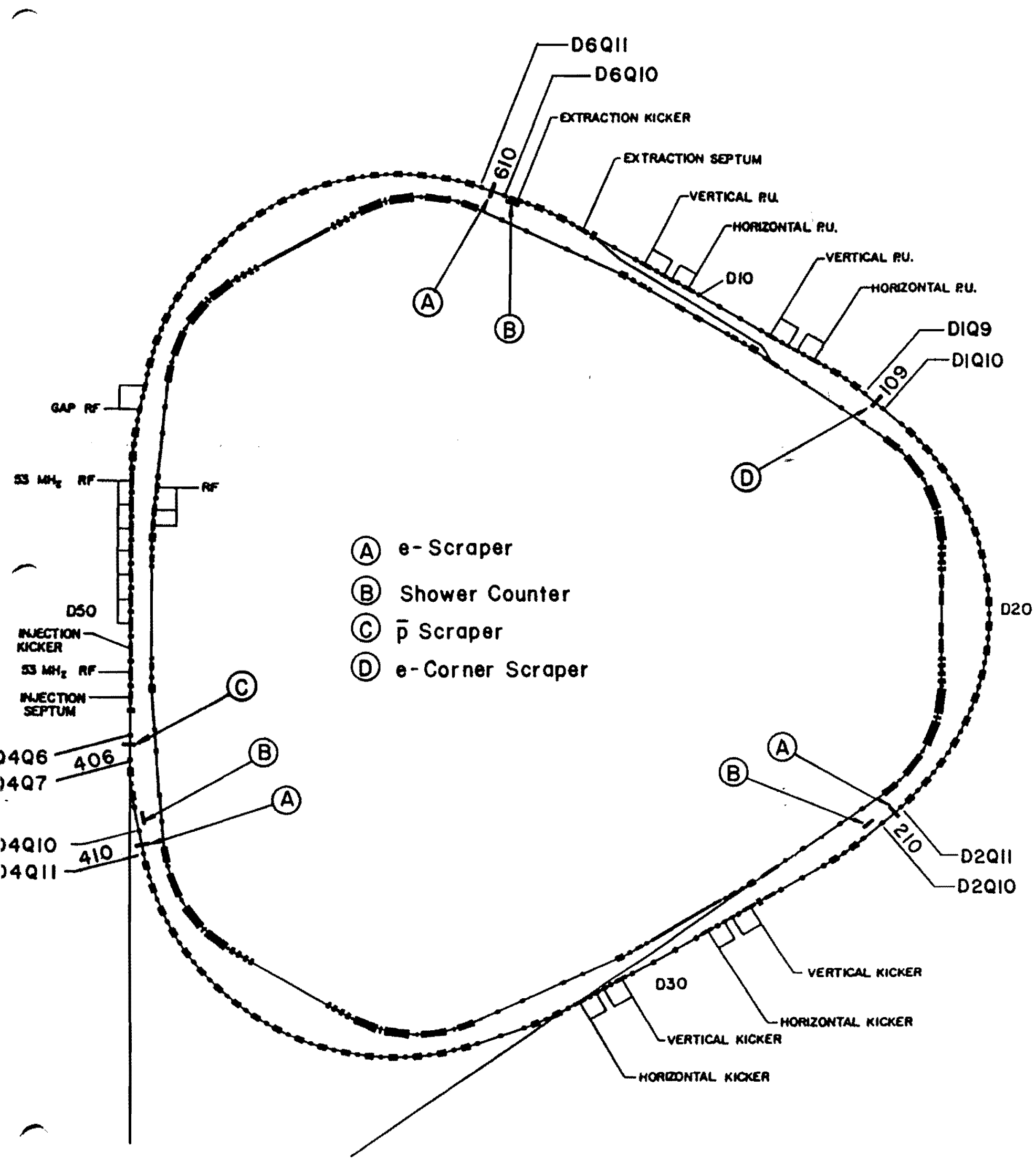

DEBUNCHER LAYOUT 
AA Electron loss signals

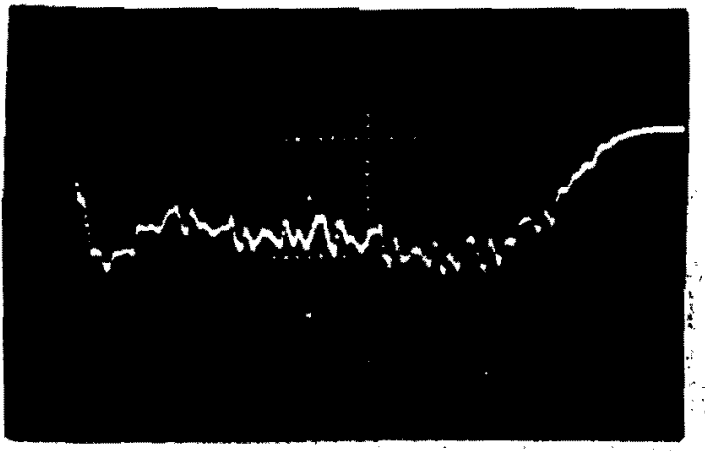

Normal operational signal.

Coherent oscillation due to mis-steering at injection

Coherent oscielatias due to $Q 71$ folie - The gradin poles steer the beam due to milaliguments

Modulation at revolution frequency clue to nijection kicker mistiming

Fig. 3 


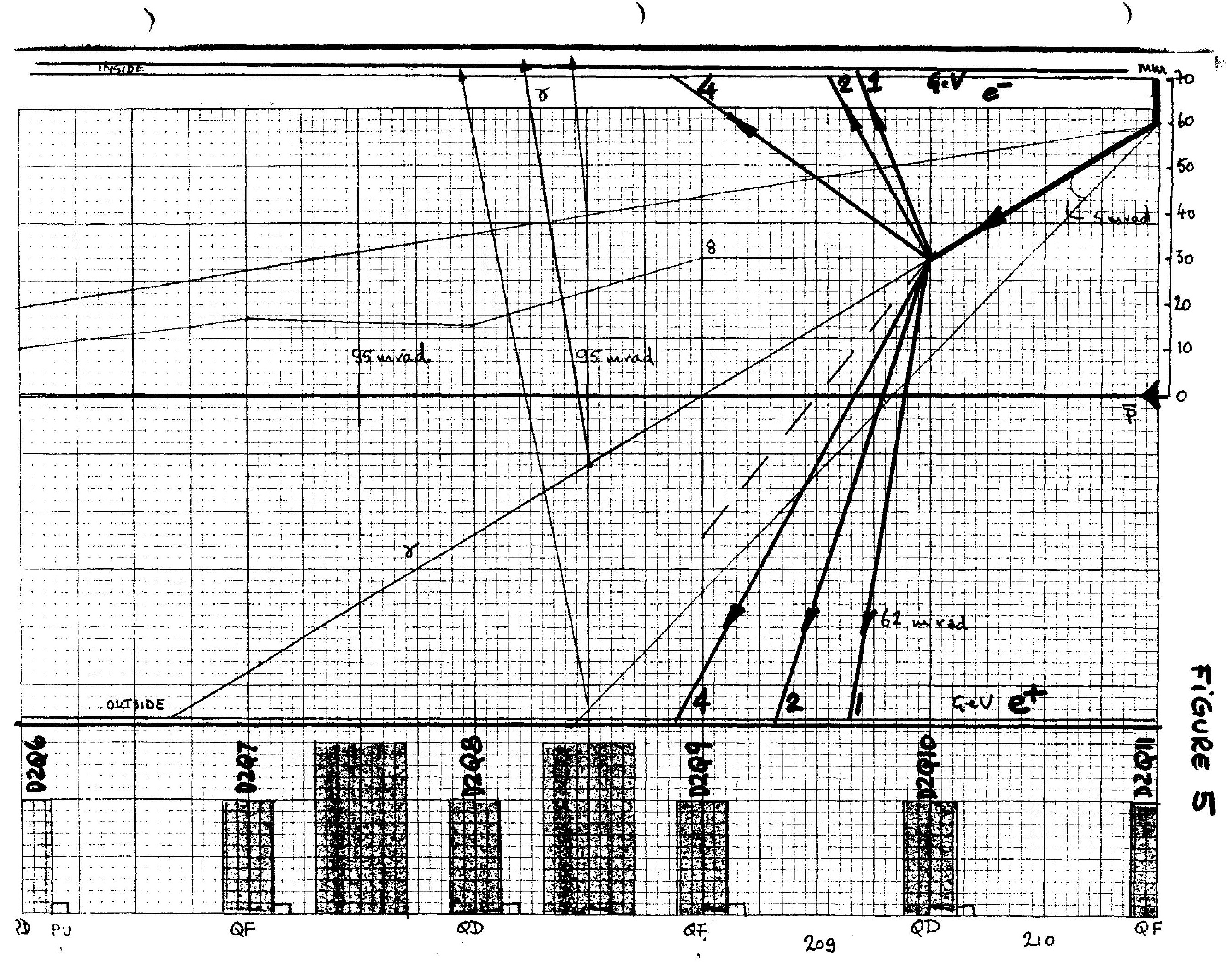




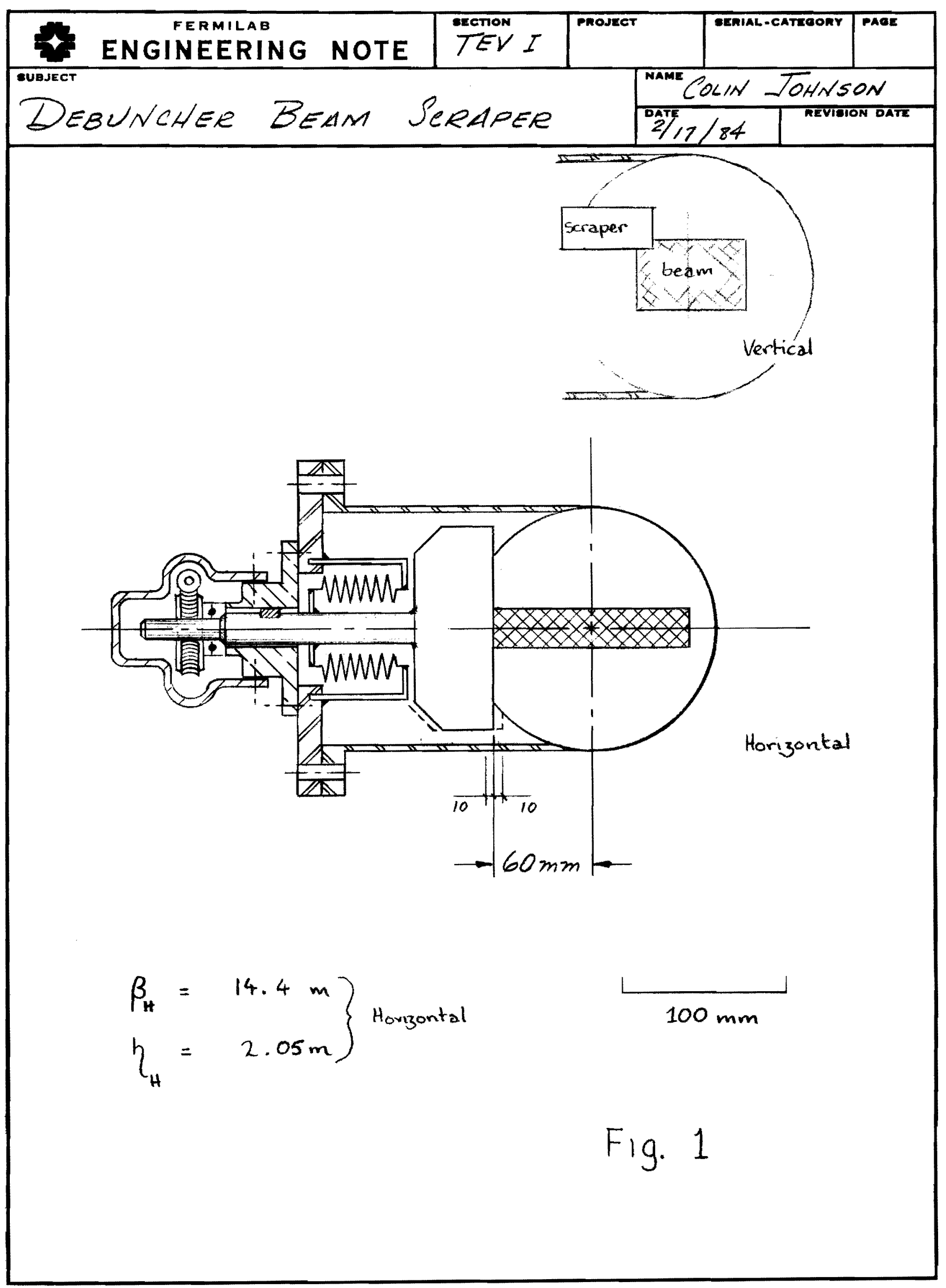




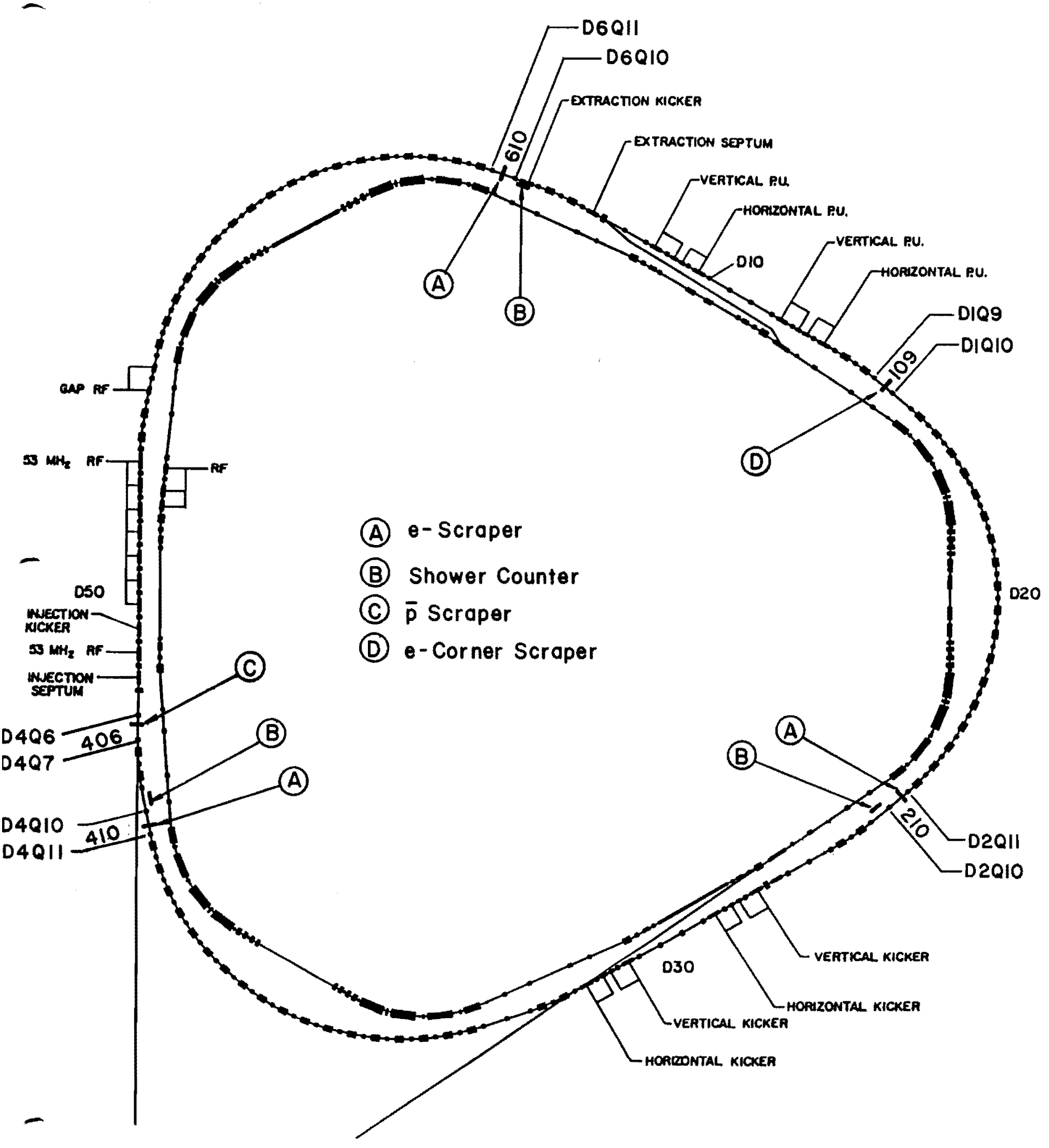

DEBUNCHER LAYOUT 
AA Electron loss signals

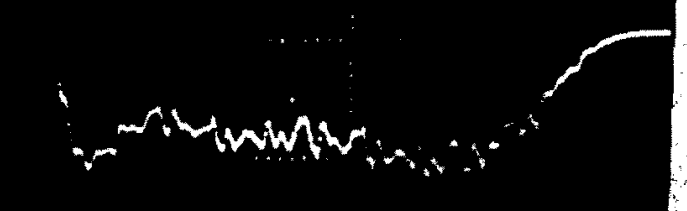

Normal operational signal.

Coherent oscillation due to mis-steering at injection

Coherent oscillations due to Q71 fuller - The quads poles steer the beam due to misalignment

Modulation at revolution frequency due to injection kicker mistiming

Fig. 3 